\title{
Genetic diversity and ISSR marker association with the quality of pineapple fiber for use in industry
}

\author{
Cintia Paula Feitosa Souza ${ }^{a}$, Claudia Fortes Ferreira ${ }^{\mathrm{b}}$, Everton Hilo de Souza ${ }^{\mathrm{a}, \mathrm{b}}$, \\ Alfredo Rodrigues Sena Neto ${ }^{\mathrm{c}, \mathrm{d}}$, José Manoel Marconcini ${ }^{\mathrm{c}}$, Carlos Alberto da Silva Ledo ${ }^{\mathrm{b}}$, \\ Fernanda Vidigal Duarte Souza ${ }^{\text {,** }}$
}

${ }^{a}$ Universidade Federal do Recôncavo da Bahia, 44380-000, Cruz das Almas, Bahia, Brazil

b Embrapa Mandioca e Fruticultura, Rua Embrapa, S/N, 44380-000, Cruz das Almas, Bahia, Brazil

c Embrapa Instrumentação, Rua XV de Novembro, n¹452, C. P. 741, 13560-970 - Centro, São Carlos, São Paulo, Brazil

d Universidade Federal de Lavras, 37200-000, Lavras, Minas Gerais, Brazil

\section{A R T I C L E I N F O}

\section{Keywords:}

Ananas comosus (L.) merr

Genetic diversity

Nonparametric tests

Spearman

Kruskal-Wallis

DNA markers

\begin{abstract}
A B S T R A C T
Interest in biodegradable products such as natural fibers for use in various industries is growing. These fibers are an inexpensive alternative with environmentally friendly appeal in comparison to other materials used. This article reports evaluation of the genetic variability of 13 pineapple genotypes to ascertain the use their fibers as mechanical reinforcement in composites applied in industry and analyzes possible association of 217 bands from 17 ISSR markers with fiber quality characteristics by nonparametric tests (Spearman correlation and KruskalWallis). The study of genetic diversity, using qualitative and quantitative data, shows there are genotypes genetically close to curauá (a close relative of pineapple), whose fibers are already commonly used in the industry. Of the 217 ISSR bands, 11 were selected based on their high correlation $\left(0.63434^{*}\right.$ to $\left.0.76169^{* *}\right)$ regarding all four variables for fiber quality, enabling identification of a set of primers which can be used in early selection of promising genotypes after sequencing and validation. The possibility of using marker assisted selection in pineapple for fiber quality will bring great returns, not only for the industry, but also to ensure the sustainability of this productive sector. This is the first report of the use of nonparametric methods to analyze fiber quality of this plant.
\end{abstract}

\section{Introduction}

There is a growing global trend toward maximization of natural resources through new products and processes, which leads to the exploitation of new materials from renewable natural resources to enhance sustainability (Erickson et al., 2012). Composites produced from polymeric matrices, reinforced with natural fibers, have been replacing manufactured fibers and present advantageous mechanical properties in addition to being environmentally friendly (Beltrami et al., 2014).

There are several studies in the literature on the use of natural fibers to reinforce composites of different plants, such as rice, ramie, jute, sisal, flax and pineapple (Mohanty et al., 2002; Romanzini et al., 2013; Sena Neto et al., 2013, 2015). Pineapple [Ananas comosus (L.) Merr.] is one of the main fruits consumed worldwide, and in Brazil is produced in all states, with planted area of approximately 61,000 ha and production of 2.48 million metric tons in 2013 (FAO, 2016).
Regarding the use of fibers as mechanical reinforcement in composites for the industry, most of the studies refer to commercial varieties, with scant attention paid to wild or hybrid varieties, whose potential for fiber is still unknown (Sena Neto et al., 2013, 2015). An exception is made for a South American variety, curauá (A. comosus var. erectifolius), whose fibers are already being used in these composites as mechanical reinforcement (Sena Neto et al., 2013, 2015; Beltrami et al., 2014). Embrapa has a large collection of the Ananas genus, with about 600 accessions (Souza et al., 2012), which can be better exploited for this purpose.

However, the specific characterizations for use of plant fibers in the development of composites are laborious, involving time, skilled labor and high cost. Thus, the development of molecular tools that can support marker assisted selection (MAS) can be of great value to increase the supply of quality fiber sources for industry. In preliminary studies, accessions from the Embrapa pineapple germplasm bank and hybrids of Curauá were subjected to fiber quality characterizations for

\footnotetext{
* Corresponding author.

E-mail address: fernanda.souza@embrapa.br (F.V.D. Souza).
} 
this purpose (Sena Neto et al., 2013, 2015).

DNA molecular methods are used to study genetic variability in plants aiming to identify and determine relationships at cultivar and species level. ISSR (Inter-Simple Sequence Repeats) markers are used in many studies since it is a simple, efficient and reproducible marker with high polymorphism (Reddy et al., 2002). These markers being highly polymorphic are appropriate for identification of genotypes (RakoczyTrojanowska and Bolibok, 2004), as well as studies of genetic diversity (Tanya et al., 2011; Noormohammadi et al., 2013; Kaya, 2015).

This study aimed to identify associations between bands from ISSR markers with the variables for fiber quality ("Onset" temperature of thermoxidative degradation; elastic modulus; cellulose crystallinity index; tensile strength) of 13 pineapple varieties (among hybrids and wild species), whose phenotyping for fiber quality had been previously conducted. The associated set of bands will be the basis for possible marker assisted selection (MAS) in the pineapple genetic breeding program (PGBP) in order to identify promising genotypes for use in the industry through further sequencing and validation. This article contains the first report of the use of nonparametric tests for selection of pineapple varieties for this purpose.

\section{Material and methods}

\subsection{Plant material}

Nine hybrids from the pineapple genetic breeding program of the Embrapa Cassava and Fruits research unit, two parental genotypes (Ananas comosus var. erectifolius and A. comosus var. bracteatus) and two bromeliad species (Hohenbergia catingae Ule and Neoglaziovia variegata Mez) were used in this study (Table 1). The characterization of fiber quality was carried out by Sena Neto et al. $(2013,2015)$ and their results were used to select the materials used in this study.

\subsection{DNA extraction and amplification}

DNA extraction was carried out from leaves of 13 pineapple genotypes using the CTAB method proposed by Doyle and Doyle (1990). The samples were diluted to $10 \mathrm{ng} \mathrm{LL}^{-1}$ and maintained at $-20{ }^{\circ} \mathrm{C}$ until use. Amplification reactions were prepared with a final volume of $25 \mu \mathrm{L}$, containing the following reagents: $50 \mathrm{mM} \mathrm{KCl}, 10 \mathrm{mM}$ Tris- $\mathrm{HCl}$ (pH 8.3), $2.5 \mathrm{mM} \mathrm{MgCl2}, 100 \mathrm{mM}$ each of dNTPs (dATP, dTTP, dGTP, dCTP), $0.2 \mathrm{mM}$ each primer, $20 \mathrm{ng}$ of genomic DNA and one unit of Taq DNA polymerase (Pharmacia Biotech, USA).

A total of 17 ISSR primers were used in the study (Table 2), and PCR amplifications were carried out in a PTC-100 thermocycler (MJ Research) using the following program: initial denaturation at $94{ }^{\circ} \mathrm{C}$ for $3 \mathrm{~min}$; 45 cycles of denaturation at $94^{\circ} \mathrm{C}$ for $45 \mathrm{~s}$, primer annealing at $48{ }^{\circ} \mathrm{C}$ for $45 \mathrm{~s}$, extension at $72{ }^{\circ} \mathrm{C}$ for $1 \mathrm{~min}$; and a final extension at
Table 2

ISSR markers used in the study of genetic diversity and nonparametric analysis.

\begin{tabular}{lllll}
\hline \multicolumn{2}{c}{ Marker name } & & Sequence & Number of band/primer \\
\cline { 1 - 2 } \cline { 4 - 5 } ISSR 07 & DiCA5'CY & & CYCACACACACACACACA & 06 \\
ISSR 24 & DiGT5'A & & AGTGTGTGTGTGTGTGT & 15 \\
ISSR 30 & TriCAC3'YC & & CACCACCACCACCACYC & 11 \\
ISSR 31 & TriCAC5'CR & & CRCACCACCACCACCAC & 13 \\
ISSR 32 & TriCAC5'CY & & CYCACCACCACCACCAC & 20 \\
ISSR 35 & TriCAG3'YC & & CAGCAGCAGCAGCAGYC & 05 \\
ISSR 37 & TriCAG5'CY & & CYCAGCAGCAGCAGCAG & 14 \\
ISSR 39 & TriGTG3'RC & & GTGGTGGTGGTGGTGRC & 11 \\
ISSR 40 & TriGTG3'YC & & GTGGTGGTGGTGGTGYC & 17 \\
ISSR 47 & TriTGT5'CY & & CYTGTTGTTGTTGTTGT & 13 \\
ISSR 57 & TriACC 3'RC & & ACCACCACCACCACCRC & 07 \\
ISSR 62 & TriAGG 3'RC & & AGGAGGAGGAGGAGGRC & 14 \\
ISSR 79 & TriCAC 3'RC & & CACCACCACCACCACRC & 09 \\
ISSR 83 & TriCTG 3'RC & & CTGCTGCTGCTGCTGRC & 10 \\
ISSR 87 & TriCGT 3'RC & & CGTCGTCGTCGTCGTRC & 15 \\
ISSR 93 & TriGAG 3'RC & & GAGGAGGAGGAGGAGRC & 12 \\
ISSR 96 & TriGTC 3'RC & GTCGTCGTCGTCGTCRC & 17 \\
& & &
\end{tabular}

$72{ }^{\circ} \mathrm{C}$ for $7 \mathrm{~min}$ and $10{ }^{\circ} \mathrm{C} \infty$ (Williams et al., 1990).

The amplification products were separated by electrophoresis in $2.5 \%$ agarose gel treated with $0.5 \mathrm{mg} \mathrm{ml}^{-1}$ of ethidium bromide and visualized in UV light transilluminator. The electrophoretic profiles of the bands were photographed using the Vilber Lourmat ${ }^{\mathrm{TM}}$ photo documentation system.

\subsection{Analysis of the electrophoretic profiles}

The 217 fragments obtained from the amplification reactions of 17 ISSR molecular markers were assessed as absent (0) or present (1). The matrix of genetic dissimilarity of the molecular data was calculated using Jaccard́s similarity coefficient (Jaccard́s complementarity index $=1-\mathrm{c}$ ). The distance matrix was calculated using the PAST software version 1.34 (Hammer et al., 2005), cluster analysis by the UPGMA method (Unweighted Pair-Group Method with Arithmetic Mean), and the validation of the cluster analysis was determined by the cophenetic correlation coefficient (CCC) (Sokal and Rolf, 1962).

\subsection{Analysis of phenotypic characteristics}

The fiber quality characteristics were evaluated by Sena Neto et al., (2013, 2015) for the same genotypes for the following variables: cellulose crystallinity index (Ic), onset temperature of thermoxidative degradation (TOOTD), tensile strength $\left(\sigma_{s}\right)$ and elastic modulus $(E)$ ] of the fibers (Table 3 ). The dissimilarity matrix was calculated for the quantitative data using the average Euclidean distance using the GENES software (Cruz, 2006), also used to perform the cluster analysis by the

Table 1

Genotypes used in the study of fiber quality and association with ISSR marker bands via nonparametric methods and multivariate analysis.

\begin{tabular}{|c|c|c|}
\hline Name & Scientific name & $\begin{array}{l}\text { Code } \\
\text { (Sena Neto et al., 2015) }\end{array}$ \\
\hline Erectifolius & Ananas comosus (L.) Merr. var. erectifolius (L.B.Sm.) Coppens \& F.Leal & Curauá \\
\hline Bracteatus & A. comosus (L.) Merr. var. bracteatus (Lindl.) Coppens \& F.Leal & BGA 110 (bract) \\
\hline Hohenbergia cantigae Ule & & Bilbergia sp. \\
\hline Neoglaziovia variegata Mez & & Neoglasiovia \\
\hline FIBROX & A. comosus var. erectifolius $\times A$. comosus var. bracteatus & Erect $\times$ Bract PL 02 \\
\hline FIBEST & A. comosus var. erectifolius $\times$ A. comosus var. bracteatus & Erect $\times$ Bract PL 01 \\
\hline FIBCER & A. comosus var. erectifolius $\times$ A. comosus var. bracteatus & $739 \times 17-\mathrm{PL} 03$ \\
\hline FIBPOT & A. comosus var. erectifolius $\times$ A. comosus var. bracteatus & $750 \times 128-\mathrm{PL} 01$ \\
\hline FIBMIN & A. comosus var. erectifolius $\times$ A. comosus var. bracteatus & $750 \times 128-$ PL02 \\
\hline FIBSAI & A. comosus var. bracteatus $\times$ A. comosus var. erectifolius & $126 \times 804-$ PL08 \\
\hline FIBNEG & A. comosus (L.) Merr. var. ananassoides (Baker) Coppens \& F. Leal $\times$ A. comosus var. erectifolius & $526 \times 804-\mathrm{PL} 01$ \\
\hline FIBBOY & A. comosus var. ananassoides $\times$ A. comosus var. erectifolius & $526 \times 804-$ PL05 \\
\hline FIBMAC & A. comosus var. comosus $\times$ A. macrodontes $\mathrm{E}$. Morren & Prima $\times$ Macrod $(716)$ \\
\hline
\end{tabular}


Table 3

Values of cellulose crystallinity index (Ic), onset temperature of thermoxidative degradation (OTTD), tensile strength ( $\sigma \mathrm{s})$ and elastic modulus $(E)$ of the leaf fibers of the studied pineapple genotypes according to Sena Neto et al. (2015).

\begin{tabular}{lllll}
\hline Name & Ic $(\%)$ & OTTD $\left({ }^{\circ} \mathrm{C}\right)$ & $\sigma_{s}(\mathrm{MPa})$ & $E(\mathrm{GPa})$ \\
\hline Neoglaziovia variegata Mez & 73.0 & 257 & 629 & 37 \\
Hohenbergia cantigae Ule & 48.7 & 249 & 212 & 15 \\
Curauá - A. comosus var. erectifolius & 64.0 & 256 & 753 & 54 \\
A. comosus var. Bracteatus & 70.8 & 240 & 828 & 75 \\
FIB-ROX & 73.5 & 252 & 1049 & 51 \\
FIB-EST & 70.3 & 258 & 1092 & 59 \\
FIB-CER & 74.7 & 261 & 1071 & 59 \\
FIB-POT & 78.7 & 264 & 1231 & 73 \\
FIB-SAI & 77.6 & 272 & 1221 & 86 \\
FIB-NEG & 61.0 & 268 & 1100 & 75 \\
FIB-BOY & 73.6 & 249 & 1309 & 76 \\
FIB-MIN & 62.9 & 248 & 891 & 65 \\
FIB-MAC & 74.7 & 254 & 832 & 50
\end{tabular}

UPGMA method. The validation of the clusters was determined by the cophenetic correlation coefficient (CCC) (Sokal and Rolf, 1962).

\subsection{Joint analysis of molecular and phenotypic data}

Joint cluster analysis of molecular and phenotypic data was performed to determine the distance matrix using the Ward-MLM method based on the Gower algorithm (1971). Validation of the clusters was determined by the cophenetic correlation coefficient (CCC) (Sokal and Rolf, 1962). The GENES software (Cruz, 2006) was used to calculate the correlation between the phenotypic and genotypic matrices using Manteĺs test with 10,000 permutations.

\subsection{Nonparametric methods}

The correlation between the four variables for fiber quality and the 217 bands from the 17 molecular markers was calculated by the Spearman and Kruskal Wallis tests (nonparametric tests) using the SAS statistical software (Sas Institute Inc., 2010).

Nonparametric tests are used when there is a small number of genotypes and no normality in the data, the most suitable for the data available in our work.

\section{Results and discussion}

\subsection{Genetic diversity based on molecular data - ISSR markers}

All 17 primers tested in the 13 genotypes generated easily detectable bands on agarose gels, providing a total of 217 bands. The number of bands per primer ranged from 5 (ISSR 35) to 20 (ISSR 35), with an average of 12 bands per primer.

The genetic distance values based on the dissimilarity matrix with the molecular data ranged from 0.09 to 0.64 . The dendrogram generated using Jaccard́s index formed four clusters (Fig. 1). G1 was formed by the $H$. catingae species; G2 by ten genotypes, eight hybrids and their parentals, A. comosus var. erectifolius and A. comosus var. bracteatus; G3 by a hybrid involving two species (A. comosus var. and $A$. macrodontes); and G4 by the species $N$. variegata. The least genetically dissimilar genotypes were the FIB-MIN and FIB-POT hybrids and the largest dissimilarities were observed between $H$. catingae and the other genotypes.

At first, the hybrids FIB-ROX, FIB-EST, FIB-CER FIB-POT, FIB-SAI, FIB-NEG, FIB-BOY and FIB-MIN, all from crosses using the reference variety "Curauá", were considered promising, since they are genetically closest, so they should be investigated with more fiber quality criteria for possible use in the industry.

\subsection{Diversity based on phenotypic characteristics}

As mentioned above, the data related to the fiber quality characteristics of the genotypes studied were obtained from those previously investigated by Sena Neto et al. (2013, 2015).

Regarding quantitative variables, the dendrogram formed two clusters by the average Euclidean distance (Fig. 1B). G1 contained $H$. catingae, a completely distinct species and probably isolated from the others due to the values of the cellulose crystallinity index $(48.7 \%)$, tensile strength (212 MPa) and elastic modulus (15 GPa) of the fibers, much lower compared to the values of other genotypes. G2 was formed by all hybrids, their parents and $N$. variegata. This cluster is explianed by the fact the varieties have close values regarding the four analyzed quantitative characteristics.

The hybrids in this study have great potential for use as mechanical reinforcement, since they showed similarity to curauá. These similarities were both genetic and in relation to the properties required of composites for mechanical reinforcement.

\subsection{Joint cluster analysis}

In order to obtain a more complete analysis, taking advantage of both molecular and phenetypic data, joint cluster analysis was carried out. This analysis, conducted with the 13 genotypes, 217 ISSR bands and four variables for fiber quality, resulted in four clusters (Fig. 1C) by the UPGMA method and using the Gower algorithm. The genetic distances ranged from 0.14 to 0.50 , where the most dissimilar genotypes were the hybrid FIB-CER and $\mathrm{H}$. cantigae and the least dissimilar were the hybrids FIB-EST and FIB-POT, as well as the hybrids FIB- POT and FIB-SAI. G1 was formed only of $H$. catingae. In the G2 group, seven hybrids and their parentals were clustered. Two hybrids, FIB-MIN and FIB-MAC, although from different genetic backgrounds, were clustered in G3 due to sharing the same values for quantitative fiber quality characteristics. Finally, G4 was formed by the species $N$. variegata.

Joint cluster analysis, which allows the combined evaluation of quantitative and qualitative data, is an effective method to identify the relationship between genotypes, maximizing their variability potential and use in genetic breeding programs (Souza et al., 2012). Regarding the cophenetic correlation coefficients, the obtained values were 0.96 , 0.87 and 0.96 for the data evaluated by Jaccard́s coefficient index, average Euclidean distance and the Gower algorithm (1971), respectively. According to Sokal and Rohlf (1962), cophenetic correlation values greater than 0.80 indicate good fit between the original distance matrices and the graphical distances, which supports the increased reliability by cluster analysis using the UPGMA method.

The results reported here are consistent with those presented by Sena Neto et al. (2013, 2015), in which the authors showed significant differences in the thermal and mechanical properties for the fiber quality in the different pineapple genotypes studied, thus providing more options for the use of these fibers for mechanical reinforcement in industrial composites.

Among the three cluster analysis algorithms used, two presented lower dissimilarity between the hybrids FIB-POT and FIB-BOY in relation to the cultivar curauá, used as best reference for fiber quality in composites in the industry. The Jaccard index and the Gower (1971) algorithm provided the most suitable interpretation of the genotypes to be better exploited based on their genetic similarities to curauá. The correlation between the phenotypic and genotypic matrices was $r=0.70, p<0.01,10,000$ permutations, based on Manteĺs test using the GENES software (Cruz, 2006).

\subsection{Nonparametric tests}

Although not expected, 11 positive and significant correlations (ranging from $0.63434^{*}$ to $0.76169^{* *}$ ) between the 217 bands and the 

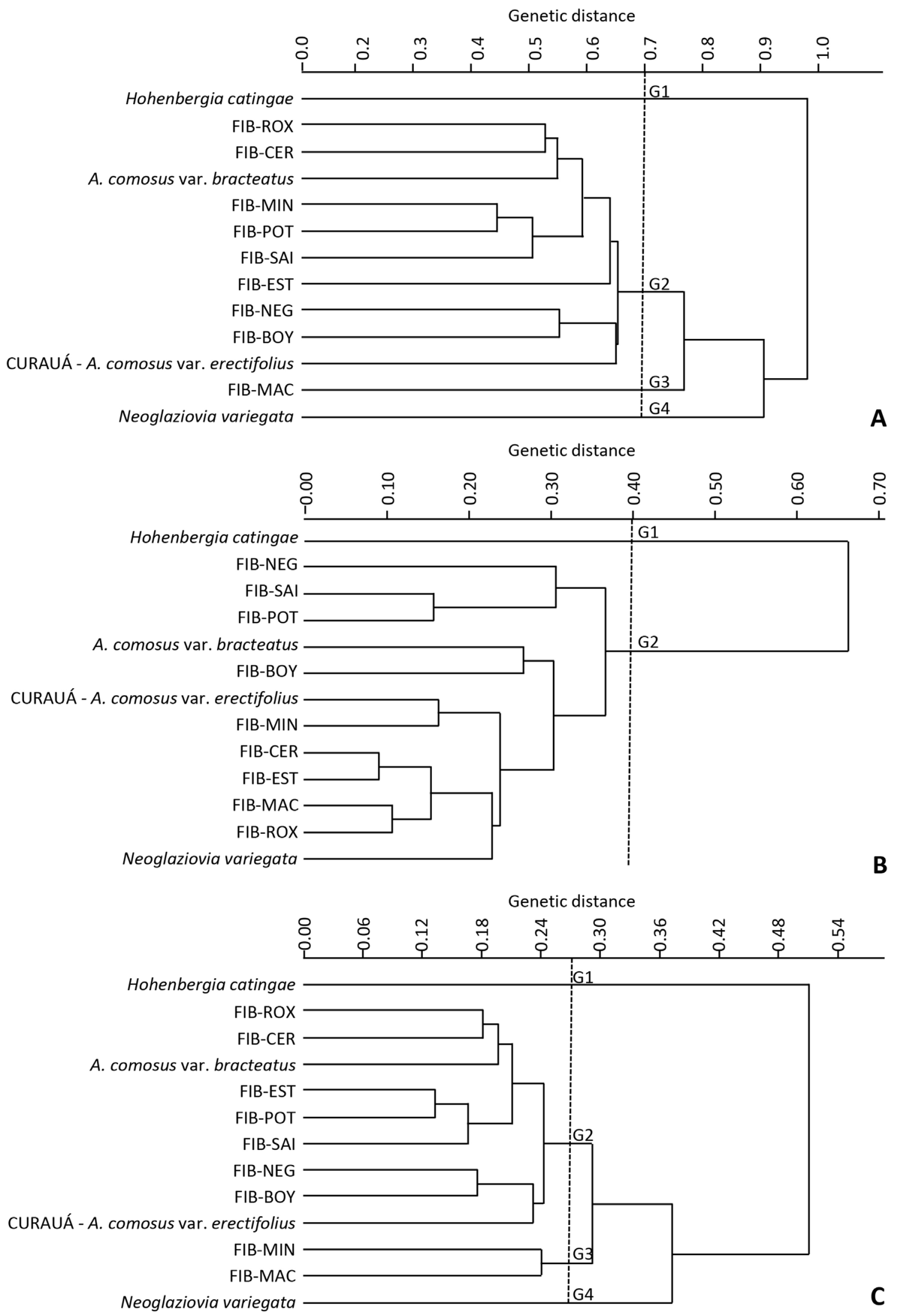

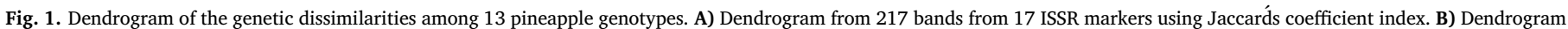

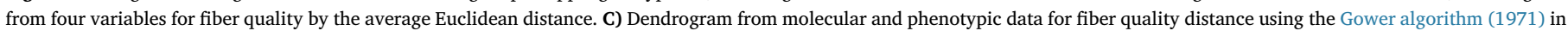
joint analysis - qualitative and quantitative data. 
Table 4

Spearman and Kruskal Wallis correlation used for linkage analysis markers and molecular variables for quality of pineapple fibers.

\begin{tabular}{|c|c|c|c|c|}
\hline Marker & Variable & Spearman & $\begin{array}{l}\chi^{2} \\
\text { align = "left" }\end{array}$ & $\begin{array}{l}\operatorname{Pr}>\chi^{2} \\
\text { align }=\text { "left" }\end{array}$ \\
\hline M124-ISSR 32 & "Onset" temperature of thermoxidative degradation (OTTOD) & $0.76169^{* * *}$ & 6.96 & 0.0083 \\
\hline M20-ISSR 31 & Elastic modulus (E) & $0.73394^{* * *}$ & 6.46 & 0.0110 \\
\hline M173-ISSR 93 & Elastic modulus (E) & $0.73354^{* * *}$ & 6.46 & 0.0110 \\
\hline M70-ISSR 40 & Cellulose crystallinity index (Ic) & $0.67705^{*}$ & 5.50 & 0.0190 \\
\hline M71-ISSR 40 & Cellulose crystallinity index (Ic) & $0.67705^{*}$ & 5.50 & 0.0110 \\
\hline M117-ISSR 32 & "Onset" temperature of thermoxidative degradation (OTOTD) & $0.66907^{*}$ & 5.37 & 0.0205 \\
\hline M197-ISSR 35 & Tensile strength $\left(\sigma_{s}\right)$ & $0.66815^{*}$ & 5.36 & 0.0206 \\
\hline M57-ISSR 79 & "Onset" temperature of thermoxidative degradation (OTTOD) & $0.64889^{*}$ & 4.63 & 0.0314 \\
\hline M130-ISSR 32 & Elastic modulus (E) & $0.64097^{*}$ & 4.93 & 0.0264 \\
\hline M173-ISSR 93 & Tensile strength $\left(\sigma_{s}\right)$ & $0.63439^{*}$ & 4.83 & 0.0280 \\
\hline M132-ISSR 32 & Tensile strength $\left(\sigma_{s}\right)$ & $0.63434 *$ & 4.83 & 0.0280 \\
\hline
\end{tabular}

* Significant at $5 \%$ probability.

** Significant at $1 \%$ probability.

four fiber quality variables previously characterized by Sena Neto et al. (2013, 2015) were observed (Table 4).

Overall, the correlations among the 217 bands from 17 ISSR markers and the four phenotypic variables for fiber quality ranged from 0.0 to $0.76 * *$. For the cellulose crystallinity index (Ic), there was a correlation with both bands, M70 and M71, for the single ISSR 40 marker, both having values of $0.67705^{*}$. For the tensile strength $\left(\sigma_{s}\right)$ variable, bands M197 (ISSR 35), M173 (ISSR 93) and the M132 band (ISSR 32) showed correlations ranging from $0.66815^{*}$ to $0.63434 *$ In the case of the elastic modulus (E), three bands, M20 (ISSR 31), M173 (ISSR 93) and M130 (ISSR 32), were correlated, with values of $0.73393^{* *}, 0.73354^{* *}$ and $0.64097^{*}$, respectively.

The highest correlation, $0.76169 * *$, between band and characteristic for fiber quality was for ISSR 32, band M124, for the variable onset temperature of thermooxidative degradation (OTTOD). Two other high correlations were obtained for the same variable for bands M117 and M57, ISSR 32 and ISSR 79 markers, respectively.

Sena Neto et al. $(2013,2015)$ reported that the onset temperature of the thermooxidative degradation is considered as a reference for the maximum working temperature to which fibers can be subjected during an industrial process, like extrusion or injection mould of composites with natural fibers. Therefore, this is a characteristic of fundamental importance and one of the main factors limiting the use of lignocellulosic fibers as reinforcement in composite materials. Since the production of composite materials usually involves processes (e.g. injection molding, thermoforming) with high temperatures, so the material can flow and acquire a specific format (Chawla, 2012).

Nonparametric tests are widely used, especially when evaluating a small number of genotypes without normality of the data (Singh et al., 2013). In this work, since 13 pineapple genotypes had already been phenotyped for four variables related to fiber quality, after genotyping via ISSR markers we attempted to evaluate possible correlation between the bands from the ISSR markers and the fiber quality characteristics by nonparametric tests.

As mentioned previously, these tests do not require replicates and do not consider normal distribution of data, so they were chosen to verify the possibility or not of associations between the bands and the characteristics of interest, since the main limitation, phenotyping for fiber quality, is a very laborious and limiting step in the case of pineapple, and had already been conducted (Sena Neto et al., 2013,2015).

Nonparametric tests are used when the data does not follow normal distribution and does not have replicates involved, like this work, and therefore were chosen to investigate any possible correlation between bands and phenotypic variation for fiber quality since both phenotypic and genotypic data were available.

It should also be mentioned that the phenotypical evaluation for pineapple fiber quality for use as composites is one of the major limitations in studying segregation of populations because the methods for this phenotyping are extremely laborious and basically two to five traits can be analyzed for a maximum of 20 pineapple samples per year, making it impossible to analyze any data on the correlation between marker and the variable of interest other than through nonparametric tests.

Although we expected no significant correlation when the data were analyzed through the Spearman and Kruskal-Wallis tests, the preliminary result showed that there was high and significant correlation, at 5 and $1 \%$ probability, between ISSR bands and variables for fiber quality, which can, after sequencing and validation, be exploited in marker assisted selection (MAS) by our pineapple breeding program (PGBP), which will be expanded to provide materials for the industry. The possibility of using MAS is the first step to quicker, low cost early identification of promising pineapple genotypes to be exploited in the PGBP. Therefore, the use of nonparametric tests for this purpose can be a viable alternative, since the phenotypic characterization for pineapple fiber quality is practically impossible when dealing with a large number of genotypes.

Nonparametric tests with Spearman correlation and Kruskal-Wallis tests were used to identify possible association between markers potentially linked to fusarium resistance in Guandu beans (Singh et al., 2013). In that study, 36 elite genotypes of Cajanus cajan with different fusarium resistance levels were evaluated by 24 SSR markers and nonparametric tests. Six of the bands were found to be significantly related to resistance, with phenotypic variability ranging from 23.7 to $56.4 \%$.

Mace et al. (2006) also used the Kruskal-Wallis test to verify linkage between SSR loci and resistance to rust and late leaf spot diseases in 22 groundnut genotypes. They identified candidate SSR as valuable for mapping rust and LLS resistance, as well as gene pyramiding.

\section{Conclusion}

In our study, eleven bands were chosen for their highly significant correlation with fiber quality variables. Therefore, after sequencing and validation, many combinations of bands can be used to increase the chances of success of MAS.

Considering the importance of this crop in Brazil and the possibility of obtaining sufficient high-quality fibers for industrial scale supply, the results of this study are promising to consolidate the pineapple fiber as reinforcement in the formulation of composites, especially for the hybrids in this study. Moreover, the possibility of having a tool to identify other promising pineapple varieties with high-quality fibers that can be used in genetic breeding contributes to the sustainability of this sector.

The nonparametric tests (Spearman and Kruskal-Wallis) identified significant associations between ISSR bands and fiber quality traits that 
can, by sequencing and validation, enhance crop improvement to ensure the sustainability of this growing industry. This is the first step to make MAS applicable in the PGBP in the near future and thus enable a breakthrough in this productive sector.

\section{Acknowledgments}

The authors thank to Embrapa Mandioca e Fruticultura for the infrastructure and technological support, Coordenação de Aperfeiçoamento de Pessoal de Nível Superior (CAPES/Embrapa announcement 001/2011 and 15/2014) for the master scholarships granted to C.P.F. Souza and Fundação de Amparo à Pesquisa do Estado da Bahia (FAPESB announcement TSC0013/2014) for financial support.

\section{References}

Beltrami, L.V.R., Bandeira, J.A.V., Scienza, L.C., Zattera, A.J., 2014. Biodegradable composites: morphological, chemical, termal and mechanical properties of composites of poly (hydroxybutyrate-co-hydroxyvalerate) with curauá fibers after exposure to simulated soil. J. Appl. Polym. Sci. 131, 1-8.

Chawla, K.K., 2012. Composite Materials: Science and Engineering. Springer Science \& Business Media.

Cruz, C.D., 2006. Programa GENES: Aplicativo Computacional Em Genética E Estatística. UFV, Viçosa.

Doyle, J.J., Doyle, J.L., 1990. Isolation of plant DNA from fresh tissue. Focus 12, 13-15. Erickson, B., Nelson, J.E., Winters, P., 2012. Perspective on opportunities in industrial biotechnology in renewable chemicals. Biotecnol. J. 7, 176-185.

Food and Agriculture Organization, FAO, 2016. Database. United States: Database. FAO/ FAOSTAT, United States. http://faostat.fao.org/ (acessed 06.12.16).

Gower, J.C., 1971. A general coefficient of similarity and some of its properties. Biometrics 27, 857-874.

Kaya, E., 2015. ISSR Analysis for determination of genetic diversity and relationship in some Turkish Olive (Olea europaea L) cultivars. Not. Bot. Horti. Agrobo. 43, 96-99.
Mace, E.S., Phong, D.T., Upadhyaya, H.D.U., Chandra, S., Crouch, J.H., 2006. SSR analysis of cultivated groundnut (Arachis hypogaea L.) germplasm resistant to rust and late leaf spot diseases. Euphytica 152, 317-330.

Mohanty, A.K., Misra, M., Drzal, L.T., 2002. Sustainable bio-composites from renewable resources: opportunities and challenges in the green materials world. J. Polym. Environ. 10, 19-26.

Noormohammadi, Z., Hasheminejad-Ahangarani, F.Y., Sheidai, M., Ghasemzadeh-Baraki, S., Alishah, O., 2013. Genetic diversity analysis in opal cotton hybrids based on SSR, ISSR, and RAPD markers. Gen. Mol. Res. 12, 256-269.

Rakoczy-Trojanowska, M., Bolibok, H., 2004. Characteristics and a comparison of three classes of microsatellite-based markers and their application in plants. Cell. Mol. Biol. Lett. 9, 221-238.

Reddy, M.P., Sarla, N., Siddiq, E.A., 2002. Inter simple sequence repeat (ISSR) polymorphism and its application in plant breeding. Euphytica 128, 9-17.

Romanzini, D., Lavoratti, A., Ornaghi, H.L., Amico, S.C., Zaterra, A., 2013. Influence of fiber contente on the mechanical and dynamics mechanical properties of glass/ramie polymer composites. Mater. Des. 47, 9-15.

Sas Institute Inc, 2010. SAS/STAT: User's Guide, v.9,2. Cary.

Sena Neto, A.R., Araujo, M.A., Souza, F.V., Mattoso, L.H., Marconcini, J.M., 2013. Characterization and comparative evaluation of termal structural, chemical, mechanical and morphological properties of six pineapple leaf fiber varieties for use in composites. Ind. Crops. Prod. 43, 529-537.

Sena Neto, A.R., Araujo, M.A.M., Barboza, R.M.P., Fonseca, A.S., Tonoli, G.H.D., Souza, F.V.D., Mattoso, L.H.C., Marconcini, J.M., 2015. Comparative study of 12 pineapple leaf fiber varieties for use as mechanical reinforcement in polymer composites. Ind. Crops. Prod. 64, 68-78.

Singh, A.K., Ral, V.P., Chand, R., Singh, R.P., Singh, M.N., 2013. Genetic diversity studies and identification of SSR markers associated with Fusarium wilt (Fusarium udum) resistance in cultivated pigeonpea (Cajunus cajan). J. Genet. 92, 273-280.

Sokal, R.R., Rohlf, F.J., 1962. The comparison of dendrograms by objective methods. Taxon 11, 33-40.

Souza, E.H., Souza, F.V.D., Costa, M.A.P.C., Costa Junior, D.S., Santos-Serejo, J.A., Amorim, E.P., Ledo, C.A.S., 2012. Genetic variation of the Ananas genus with ornamental potential. Genet. Resour. Crop. Evol. 59, 1357-1376.

Tanya, P., Taeprayoon, P., Hadkam, Y., Srinives, P., 2011. Genetic diversity among Jatropha and Jatropha-related species based on ISSR Markers. Plant Mol. Biol. Rep. $29,252-264$.

Williams, J.G.K., Kubelik, A.R., Livak, K.J., 1990. DNA polymorphism amplified by arbitrary primers are useful as genetic markers. Nucleic Acids Res. 18, 6531-6535. 